\title{
Spinal obstruction-related versus craniocervical junction related syringomyelia: a comparative study of spinal cord injury
}

\section{yuan chenghua}

Xuanwu Hospital Department of Neurosurgery

jian guan

Xuanwu Hospital Department of Neurosurgery

yueqi du

Xuanwu Hospital Department of Neurosurgery

zeyu fang

Xuanwu Hospital Department of Neurosurgery

xinyu wang

Xuanwu Hospital Department of Neurosurgery

qingyu yao

Xuanwu Hospital Department of Neurosurgery

can zhang

Xuanwu Hospital Department of Neurosurgery

shanhang jia

Xuanwu Hospital Department of Neurosurgery

zhenlei liu

Xuanwu Hospital Department of Neurosurgery

kai wang

Xuanwu Hospital Department of Neurosurgery

wanru duan

Xuanwu Hospital Department of Neurosurgery

xingwen wang

Xuanwu Hospital Department of Neurosurgery

zuowei wang

Xuanwu Hospital Department of Neurosurgery

hao wu

Xuanwu Hospital Department of Neurosurgery

zan chen

Xuanwu Hospital Department of Neurosurgery

fengzeng jian ( $\sigma_{\text {jianfengzeng@xwh.ccmu.edu.cn ) }}$ 
Xuanwu Hospital Capital Medical University https://orcid.org/0000-0001-7860-278X

\section{Research Article}

Keywords: Syringomyelia, Decompression, Spinal cord injury, Chiari malformation, CSF

Posted Date: November 18th, 2021

DOI: https://doi.org/10.21203/rs.3.rs-1073541/v1

License: (c) (i) This work is licensed under a Creative Commons Attribution 4.0 International License. Read Full License 


\section{Abstract \\ Background}

No prior reports have focused on spinal cord injury (SCl) characteristics or inflammation after destruction of the blood-spinal cord barrier by syringomyelia. To compare the difference of syringomyelia-related central SCI between craniocervical junction (CCJ) and post-traumatic syringomyelia (PTS) before and after decompression.

\section{Methods}

Between 2015 and 2019, 106 CCJ, 26 CCJ revision and 15 PTS patients (mean history of symptoms $71.5 \pm 94.3,88.9 \pm 85.5$ and $32.3 \pm 48.9$ months). The symptom courses were analysed with the ASIA, Klekamp and Samii scoring systems and Kaplan-Meier statistics for neurological changes. The mean follow-up was $20.7 \pm 6.2,21.7 \pm 8.8$ and $34.8 \pm 19.4$ months.

\section{Results}

Compared with the other group, the interval time after PTS was longer, but the natural history of syringomyelia was shorter $(P=0.0004,0.0173$, respectively). The initial symptoms were usually paraesthesia $(P=0.258)$, and the symptoms were mainly hypoesthesia $(P=0.006)$, abnormal muscle strength $(P=0.004)$, gait $(P<0.0001)$ and abnormal urination $(P<0.0001)$. SCl associated with $P T S$ was more severe than that $C C J$ related $(P=0.003)$. The cavities in the PTS group were primarily located at the thoracolumbar level, which was different from those in the cervical-thoracic segment at the CCJ. The rate of syrinx/cord was more than $75 \%(P=0.009)$, and the intradural adhesions tended to be more severe $(P<0.0001)$. However, there were no significant differences in peripheral blood inflammation markers (PBIM) or long-term clinical efficacy except for the RBC $(P=0.042)$.

\section{Conclusion}

The natural history of PTS tends to progress faster and is more severe than CCJ related. PBIM had no distinguishing effect on the difference in inflammation of syringomyelia except for the RBC. The predictive value of NLR for syringomyelia-related inflammation except in the acute phase was negative.

\section{Introduction}

The most common clinical manifestation of syringomyelia is dilatation of the central canal of the spinal cord. It is often associated with Chiari malformation (CM), basilar invagination or atlantoaxial dislocation, arachnoid adhesion and other cerebrospinal fluid circulation disorders ${ }^{1-5}$ and is often 
associated with other spinal deformities. Thus, it is a type of chronic central spinal cord injury $(\mathrm{SCl})^{6}$. In patients with $\mathrm{SCl}$ caused by trauma, $50 \%$ of them have syringomyelia ${ }^{7,8}$. In some cases, the cavity can be reduced by intradural decompression ${ }^{9}$. However, SCl-related symptoms, such as dissociative sensory disturbances, muscle atrophy, and joint deformity, are often unimproved and can even worsen ${ }^{1}$. Therefore, how to repair SCl caused by the cavity is a bottleneck in clinical treatment. Ependymal cells surrounding the central canal are a source of endogenous stem cells, indicating a potential method of endogenous $\mathrm{SCl}$ repair ${ }^{10,11}$.

To date, there is no feasible animal model of $\mathrm{CM}$ except compression ${ }^{12,13}$. SCl is accompanied not only by damage to the nerve tissue-cerebrospinal fluid barrier but also by damage to the nerve tissue-blood barrier ${ }^{14}$. Therefore, the pro-oxidation and antioxidation processes that occur in the CNS may be reflected in the components of the CSF and blood. A better understanding of the potential molecular pathways associated with syringomyelia formation will reveal targets for the treatment and prevention of syringomyelia.

However, no previous reports have been published comparing syringomyelia associated with CM, Revision and PTS.

\section{Methods}

The study was reviewed and approved by the local ethics committee with waiver of informed consent from patients given its retrospective nature.

Between January 2015 and December 2019, 146 consecutive patients with intradural decompression for syringomyelia associated with CM, revision or PTS were treated at our institution (Table 1). In this study, PTS was defined as local arachnoid obstruction. The detailed inclusion and exclusion criteria are shown in Figure 1.

Klekamp and Samii scores (KS scores) ${ }^{15}$ and ASIA (evaluated by YCH) were used to evaluate the clinical course of the different groups before and after surgery. The long-term results were summarized with Kaplan-Meier statistics (Figure 2). The SC tension group was defined as $>75 \%, 50-75 \%, 25 \%-50 \%$, $10 \%-25 \%$ and $<10 \%$ by the ratio of syrinx/cord (Figure 3 ). Peripheral blood inflammation markers (PBIM) were often tested one day before surgery (Figure 4).

Patients in the $\mathrm{CM}$ and revision groups will suffer from former magnum and foramen of Magendie dredging (FMMD), as has been reported previously (Figure 5) ${ }^{9}$. Finally, for PTS patients, we adopted anterior or posterior decompression. In addition, some authors have suggested an anatomy-based comprehensive classification of spinal osteotomies or arachnoid lysis (Figure 5) for compression fractures ${ }^{8}$. 
Follow-up data were obtained during outpatient visits or by telephone interviews. Treatment success was defined as a sustained improvement of preoperative symptoms or stabilization of previously progressing symptoms. Treatment failure was defined as postoperative neurological deterioration. Patients were assessed at 3 months and 12 months postoperatively for neurological function using KS scores (Table 2). Long-term results were summarized with Kaplan-Meier statistics in the three groups. The patients also underwent postoperative MRI to determine the tension of the syrinx.

\section{Statistics}

For statistical tests of significance, the chi-square test, Kruskal-Wallis test, Mann-Whitney test, one-way ANOVA test and Fisher tests were used. Long-term follow-up was analysed with the Kaplan-Meier method by RStudio Version 1.3 to determine the rates of patients with and without postoperative clinical recurrences. For statistical analyses, the software packages Prism version 7.0 and SPSS version 25.0 were used.

\section{Results}

The clinical characteristics of the cases are presented in Table 1. None of the patients in the $\mathrm{CM}$ or revision group had an atlantoaxial dislocation history. Two patients in the $\mathrm{CM}$ group suffered from dorsal kyphosis. In one case in the CM group, syringomyelia progressed to the medulla oblongata acutely, and the syringomyelia was partially relieved after $\mathrm{FMMD}^{7}$. Interestingly, the preoperative NLR of the patient was as high as 6.5. Most patients suffered a history of trauma in the subarachnoid compression group, among which one had local subarachnoid adhesion.

The CM group had 106 patients (with a mean age of $48.0 \pm 12.7)$. The revision group $(47.0 \pm 11.3)$ was similar to the CM group. Most of patients in all three groups were concentrated in the range of 40-60 years old, but in our centre, paediatric patients are frequently treated, so the number of patients in the $\mathrm{CM}$ group aged 1-20 years was lower in this study. PTS patients were mostly male $(P<0.0001)$, and there was no significant difference in age compared with the other groups $(P=0.8018)$. Nearly half of the PTS group had experienced a complete $\mathrm{SCl}$. Compared with the revision group, the interval time after PTS was longer $(P=0.0004)$ but the natural history of syringomyelia was shorter $(P=0.0173)$. The initial symptoms of syringomyelia were usually paraesthesia $(P=0.258)$ and neuropathic pain $(13.33 \%)$, but these symptoms were rare in the PTS group. The symptoms in the PTS group were mainly hypoesthesia $(P=0.006)$, abnormal muscle strength $(P=0.004)$, abnormal gait $(P<0.0001)$ and abnormal urination $(P<0.0001)$. Compared to the other groups, the revision group had a higher rate of occipital pain $(P=0.099)$ and swallowing dysfunction $(P=0.01)$, while differences in neuropathic pain $(P=0.178)$ and dysesthesia $(P=0.303)$ showed no significance.

The cavities in the PTS group were primarily located at the thoracolumbar level, which was different from those in the cervical-thoracic segment at the craniocervical junction (CCJ). The tension in the revision 
group was more than $75 \%(P=0.009)$.

SCl associated with PTS was more severe than that associated with CCJ. Compared with the PTS group, the $\mathrm{SCl}$ caused by syringomyelia associated with the CCJ was more distributed in grade $D(P=0.003)$. Moreover, the decrease in pinprick and light touch sensation was higher in the PTS group $(P=0.0005$, $\mathrm{P}<0.0001$, respectively). However, the SCl history in the PTS group often caused irreversible damage to SC function. Although the history of the revision group was longer, there was no significant difference in ASIA compared with the CM group. There was no significant difference in UE muscle strength among the three groups $(P=0.1012)$. It should be noted that previous $S C l$ in the PTS group usually does not affect UE muscle strength. The subdural adhesions were often worse $(\mathrm{P}<0.0001)$ in the PTS group. However, there was no significant difference among the groups for PBIM except the RBC, which showed marginal statistical significance $(P=0.0421)$, presumably because the blood-SC barrier limited the reflection of the difference of chronic inflammation or the sample size is too small.

Compared with the CM group, the revision group had a higher proportion of cerebellar tonsil manipulation, but there was no significant difference $(P=0.276)$. The PTS group had the highest rate of adhesion lysis, followed by fusion. After FMMD with or without revision, complications within 7 days were observed in $23.07 \%$ and $9.43 \%$, respectively, without a significant difference for patients with PTS $(P=0.133)$. Syringomyelia declined to $58.8 \%$ of the $C M$ group and remained stable at $39.95 \%$. The rate of postoperative increase was meagre, at $1.25 \%$. Due to the higher tension of the syrinx in patients in the revision group and PTS group, the rate of syrinx cavities decrease was higher in this group, without reaching statistical significance $(P=0.123)$. The analysis of long-term outcomes suggested no significant differences among the CM group, revision group and PTS group ( $\mathrm{P}=0.257)$ (Figure 2).

Due to the influence of intradural manipulation, the relief rate of headache was low in the short term, but the rate of improvement was higher in the CM group after 3 months ( $70 \%$ vs. $41.6 \%)$. In terms of neurogenic pain, the improvement rate of the CCJ group was higher than that of the PTS group $(55.2 \%$, $52.6 \%$ vs. $42.9 \%$ ). In terms of paraesthesia, the improvement rate of the CM group and the PTS group was higher than that of the revision group ( $67.5 \%, 58.3 \%$ vs. $45.4 \%)$. For hypoesthesia, the improvement rate of the PTS group was higher than that of the CCJ group (53.8\% vs. 33.3\%, 45.4\%). Furthermore, because of the longer history in the revision group, their symptoms were often more severe. Although the lower limb symptoms in the PTS group were more severe in terms of MS, the improvement rate of MS related to cavitation was slightly lower than that in the CCJ group (38.5\% vs. $40 \%, 38.9 \%)$. In terms of gait, the improvement rate of the CM group was higher than that of the revision group and the PTS group (54.8\% vs. $28.5 \%, 23.1 \%)$, but the PTS group's past trauma history can easily lead to residual gait disorder, which makes confounding factors unable to be ruled out. In terms of urination, the improvement rate of the revision group and the PTS group was higher than that of the CM group (66.7\%, $40 \%$ vs. $33.3 \%$ ), but the proportion of urination disorders in the $\mathrm{CM}$ group and the revision group was relatively low. The CCJ group had a higher remission rate of cranial nerve symptoms in the posterior group $(72.7 \%$, $66.6 \%$ ), while in the PTS group, there were 2 cases of bulbar cavity causing related symptoms, and 1 case 
was relieved after surgery. In terms of sweating symptoms, the remission rate in the CCJ area was lower $(14.3 \%, 0 \%)$, while there were 2 patients in the PTS group, of which 1 case was relieved after surgery.

\section{Discussion}

With the ageing of society, an increasing number of cervical degenerative diseases patients suffered central $\mathrm{SCl}$, many of whom have symptoms that are more severe in the upper limbs than in the lower $\operatorname{limbs}^{16}$. Syringomyelia is an expansion of the central canal of the SC, which is the simplest form of central SCl. With the help of a syringomyelia model, we can better study central SCl caused by various conditions. $\mathrm{CM}$ is the most common clinical cause of syringomyelia. Spinal obstruction-related syringomyelia is similar to the compression syringomyelia model ${ }^{13}$. The long-term natural history of syringomyelia remains unclear ${ }^{7,17}$. The history of syringomyelia related to the craniocervical junction, especially in the revision group, was significantly longer than that in the PTS group. We suspect that the duration of the natural history may be related to the extent of SCl. In addition, most patients in these three groups suffered intradural decompression. Therefore, we made relevant clinical comparisons among the three to compare their similarities and differences to improve our understanding of central SCl. In future studies, we will explore its molecular mechanism, deepen the understanding of ependymal cells involved in the repair of $\mathrm{SCl}$, and provide a theoretical basis for endogenous $\mathrm{SC}$ repair.

In our study, PTS were mostly male. This might be because men are more likely to be injured. Studies have pointed out that the fluid in the cavity mainly comes from the subarachnoid space ${ }^{18}$. In a case of abnormal pulsation of cerebrospinal fluid, it can enter the central canal of the SC through the perivascular space. In our data, the proportion of high-tension cavitation in the PTS group was higher than that in the $\mathrm{CM}$ group, but the data of the revision group may be influenced by outpatient selection bias. The potential cause is that the mechanisms of the formation and the postoperative changes of the cavity in the PTS and $\mathrm{CM}$ groups are different, including more serious subdural adhesion and BBB destruction in PTS ${ }^{19}$. However, some authors suggest that there is no correlation between tension and injury ${ }^{20}$. With the expansion of the central canal, $\mathrm{SCl}$ and dysfunction gradually became aggravated, which is also in line with the clinical cavities in the CM group. In the CM group, the history of related symptoms lasted longer than that in the PTS group, and the progression of SCl was slower. Generally, pain-temperature crossfibres immediately in front of the central canal are the first to be involved, and the typical clinical manifestations of segmental pain-temperature sensation and tactile separation appear. Our data showed that there was no significant difference in upper limb muscle strength among the three groups, while the proportion of hypoesthesia in the revision and PTS groups was higher. In addition, we noticed that both pain and light sensation often declined in the PTS group, while a higher proportion of dissociated sensory loss was observed in the CM group, which suggests that PTS is associated with more severe trauma. However, neurogenic pain was rare in the PTS group, which suggests that central canal dilatation was more likely to be accompanied by SC parenchyma damage in the PTS group. The PTS group may be related to the faster progression of abnormal CSF circulation dysfunction; further enlargement of the central canal involves the anterior horn neurons and manifestations such as muscle atrophy. In clinical 
practice, we also noticed that in the craniocervical junction group, the first and second interosseous muscles of the upper limb or small interosseous muscle atrophy and ulnar finger extension difficulty were common. However, due to a short medical history, the PTS group seldom showed anterior motor horn injury of the upper limb or muscle atrophy. Longitudinal conduction tracts farther away from the central canal, such as the corticospinal tract and spinothalamic tract, always show signs of damage in the later stages of the disease. The revision group had a higher proportion of impaired motor function and gait than the CM group, which also supports this view. However, many studies have found that the size of syringomyelia is not related to the severity of clinical symptoms ${ }^{20,21}$. Our previous basic research found that $\mathrm{SCl}$ and changes occurred in the early stage of cavity formation. The occurrence of $\mathrm{SCl}$ caused by cavities may not be solely due to central canal expansion, but it is likely that central canal expansion and $\mathrm{SCl}$ coexist ${ }^{13,19}$. Therefore, it is necessary to further clarify the pathological damage and the mechanism of $\mathrm{SCl}$ caused by syrinx.

Generally, it has been suggested that immunity and inflammation play major roles in the initiation and development of pancreatic cancer, hepatocellular carcinoma, glioma and other tumours ${ }^{22-24}$. It has been shown that inflammation is related to changes in peripheral blood leukocytes that are related to the $\mathrm{NLR}^{25}$. The degree of preoperative PBIM, for example, neutrophils, lymphocytes, monocytes, or their ratios, has been suggested to be related to the prognosis and immunity therapy outcome of cancers $^{26,27,28}$. Ependymal cells are activated after $\mathrm{SCl}$, and then cell proliferation, differentiation and migration play a repair role ${ }^{29}$. In previous studies, we found that the number of ependymal cells increases significantly after the formation of syringomyelia. In addition, syringomyelia was found to be significantly related to the inflammatory pathway through previous syringomyelia animal model SC tissue transcriptome and metabolomic ${ }^{30}$. Here, our study confirmed the negative predictive value of the leukocyte count and NLR for inflammation. We noticed that there was a patient with acute syringomyelia progression in the CM group with a lumbar compression fracture, and his NLR was as high as 6.5, which may indicate that the inflammatory reaction for the acute phase was more severe ${ }^{7}$.

Some researchers have suggested that oxidative stress plays important roles in the pathophysiology of not only acute $\mathrm{SCl}$ but also chronic $\mathrm{SCl}^{31,32}$. Erythrocytes have been shown to be potential markers for the diagnosis of some diseases ${ }^{33}$. Some authors have suggested that erythrocytes lose all of their organelles when they mature, causing a reduction in their potential to replace proteins that have lost their functions, which makes them prone to any aberrations and very sensitive to oxidative stress ${ }^{34}$. Woźniak suggested that higher lipid peroxidation will increase the concentrations of thiobarbituric acid reactive substances in the RBCs of cervical SCl patients ${ }^{35}$. Recent studies have suggested erythrocytes as a potential biomarker in the treatment of oxidative stress-associated diseases, such as chronic obstructive pulmonary disease, cardiorespiratory fitness in chronic $\mathrm{SCl}$ individuals ${ }^{36}$ and primary open-angle glaucoma ${ }^{37}$. Some authors have already shown that mild anaemia or low RBC levels can be found after $\mathrm{SCl}^{38}$. However, other authors suggested abnormally low levels of RBCs in early chronic SCl patients and augmentations over 
time, and the levels of RBCs, $\mathrm{Hb}$ and $\mathrm{Ht}$ returned to near normal levels in late chronic $\mathrm{SCl}$ patients ${ }^{39}$. The molecular mechanism of syringomyelia needs further research to elucidate.

\section{Conclusions}

The natural history of PTS tends to progress faster. The first symptom is usually paraesthesia, and SCl is more serious than syringomyelia associated with the craniocervical junction. The difference in inflammation of syringomyelia caused by different aetiologies cannot be found through PBIM except for the RBC.

\section{Declarations}

\section{Acknowledgments}

We would like to thank AJE for the English language editing.

Funding (information that explains whether and by whom the research was supported): Not applicable Conflicts of interest/Competing interests (include appropriate disclosures) : Not applicable

Availability of data and material (data transparency) : Not applicable

Code availability (software application or custom code) : Not applicable

Ethics approval (include appropriate approvals or waivers) : The study was reviewed and approved by the local ethics committee with waiver of informed consent from patients given its retrospective nature.

Consent to participate (include appropriate statements) : Not applicable

Consent for publication (include appropriate statements) : Not applicable

Authors' contributions (All authors must be mentioned) : Chenghua Yuan: Writing - Original, Draft Data Curation, Jian Guan: Writing- Reviewing and Editing, Visualization, Yueqi Du: Software, Draft Data Curation, Zeyu Fang: Software, Draft Data Curation, Xinyu Wang: Software, Draft Data Curation, Qingyu Yao: Software, Draft Data Curation Can Zhang: Software Shanhang Jia: Resources Kai Wang: Resources Zhenlei Liu: Methodology, Resources, Wanru Duan: Resources Xingwen Wang: Resources, Visualization Zuowei Wang: Resources, Visualization Hao Wu: Resources Zan Chen: Resources Fengzeng Jian: WritingReviewing and Editing, Project administration.

\section{References}


1. Yuan C, Guan J, Du Y et al (2020) Repeat Craniocervical Decompression in Patients with a Persistent or Worsening Syrinx: A Preliminary Report and Early Results. World Neurosurg 138:e95-e105

2. CreveCoeur TS, Yahanda AT, Maher CO et al (2021) Occipital-Cervical Fusion and Ventral Decompression in the Surgical Management of Chiari-1 Malformation and Syringomyelia: Analysis of Data From the Park-Reeves Syringomyelia Research Consortium. Neurosurgery 88(2):332-341

3. Jian FZ, Chen Z, Wrede KH, Samii M, Ling F (2010) Direct posterior reduction and fixation for the treatment of basilar invagination with atlantoaxial dislocation. Neurosurgery 66(4):678-687

4. Guan J, Yuan C, Zhang C et al (2021) A novel classification and its clinical significance in Chiari I malformation with syringomyelia based on high-resolution MRI. Eur Spine J.

5. Yuan C, Yao Q, Cheng L et al (2021) Prognostic factors and nomogram prediction of survival probability in primary spinal cord astrocytoma patients. $J$ Neurosurg Spine.:1-12

6. Divi SN, Schroeder GD, Mangan JJ et al (2019) Management of Acute Traumatic Central Cord Syndrome: A Narrative Review. Global Spine J 9(1 Suppl):89S-97S

7. Yuan C, Guan J, Jian F (2020) Rapid progression of acute cervical syringomyelia: A case report of delayed complications following spinal cord injury.J Spinal Cord Med::1-5

8. Klekamp J (2012) Treatment of posttraumatic syringomyelia. J Neurosurg Spine 17(3):199-211

9. Guan J, Yuan C, Zhang C et al (2020) Intradural Pathology Causing Cerebrospinal Fluid Obstruction in Syringomyelia and Effectiveness of Foramen Magnum and Foramen of Magendie Dredging Treatment. World Neurosurg.

10. Llorens-Bobadilla E, Chell JM, Le Merre P et al (2020) A latent lineage potential in resident neural stem cells enables spinal cord repair. Science 370:6512

11. Stenudd M, Sabelstrom H, Frisen J (2015) Role of endogenous neural stem cells in spinal cord injury and repair. JAMA Neurol 72(2):235-237

12. Takano T, Uno M, Yamano T, Shimada M (1994) Pathogenesis of cerebellar deformity in experimental Chiari type I malformation caused by mumps virus. Acta Neuropathol 87(2):168-173

13. Lee JY, Kim SW, Kim SP et al (2017) A rat model of chronic syringomyelia induced by epidural compression of the lumbar spinal cord. J Neurosurg Spine 27(4):458-467

14. Berliner J, Hemley S, Najafi E, Bilston L, Stoodley M, Lam M (2020) Abnormalities in spinal cord ultrastructure in a rat model of post-traumatic syringomyelia. Fluids Barriers CNS 17(1):11

15. Klekamp J (2012) Surgical treatment of Chiari I malformation-analysis of intraoperative findings, complications, and outcome for 371 foramen magnum decompressions. Neurosurgery 71(2):365380

16. Yelamarthy PKK, Chhabra HS, Vaccaro A et al (2019) Management and prognosis of acute traumatic cervical central cord syndrome: systematic review and Spinal Cord Society-Spine Trauma Study Group position statement. Eur Spine J 28(10):2390-2407

17. Yuan C, Yao Q, Zhang C, Jian F (2019) Spontaneous resolution of syringomyelia with a 16-year serial magnetic resonance imaging follow-up: A case report and literature review. World Neurosurg. 
18. Heiss JD, Jarvis K, Smith RK et al (2019) Origin of Syrinx Fluid in Syringomyelia: A Physiological Study. Neurosurgery 84(2):457-468

19. Hemley SJ, Tu J, Stoodley MA (2009) Role of the blood-spinal cord barrier in posttraumatic syringomyelia. J Neurosurg Spine 11(6):696-704

20. Awai L, Curt A (2015) Preserved sensory-motor function despite large-scale morphological alterations in a series of patients with holocord syringomyelia. J Neurotrauma 32(6):403-410

21. Hatem SM, Attal N, Ducreux D et al (2010) Clinical, functional and structural determinants of central pain in syringomyelia. Brain 133(11):3409-3422

22. Coussens LM, Werb Z (2002) Inflammation and cancer. Nature 420(6917):860-867

23. Habtezion A (2015) Inflammation in acute and chronic pancreatitis. Curr Opin Gastroenterol 31(5):395-399

24. Michelson N, Rincon-Torroella J, Quinones-Hinojosa A, Greenfield JP (2016) Exploring the role of inflammation in the malignant transformation of low-grade gliomas. J Neuroimmunol 297:132-140

25. Capone M, Giannarelli D, Mallardo D et al (2018) Baseline neutrophil-to-lymphocyte ratio (NLR) and derived NLR could predict overall survival in patients with advanced melanoma treated with nivolumab. J Immunother Cancer 6(1):74

26. Bilen MA, Martini DJ, Liu Y et al (2019) The prognostic and predictive impact of inflammatory biomarkers in patients who have advanced-stage cancer treated with immunotherapy. Cancer 125(1):127-134

27. Pierscianek D, Ahmadipour Y, Oppong MD et al (2019) Blood-Based Biomarkers in High Grade Gliomas: a Systematic Review. Mol Neurobiol 56(9):6071-6079

28. Chen M, Zheng SH, Yang M, Chen ZH, Li ST (2018) The diagnostic value of preoperative inflammatory markers in craniopharyngioma: a multicenter cohort study. J Neurooncol 138(1):113122

29. Fan B, Wei Z, Yao X et al (2018) Microenvironment Imbalance of Spinal Cord Injury. Cell Transplant 27(6):853-866

30. Mohrman AE, Farrag M, Huang $\mathrm{H}$ et al (2017) Spinal Cord Transcriptomic and Metabolomic Analysis after Excitotoxic Injection Injury Model of Syringomyelia. J Neurotrauma 34(3):720-733

31. Fatima G, Sharma VP, Das SK, Mahdi AA (2015) Oxidative stress and antioxidative parameters in patients with spinal cord injury: implications in the pathogenesis of disease. Spinal Cord 53(1):3-6

32. Hall ED (2011) Antioxidant therapies for acute spinal cord injury. Neurotherapeutics 8(2):152-167

33. Crawford JH, Chacko BK, Kevil CG, Patel RP (2004) The red blood cell and vascular function in health and disease. Antioxid Redox Signal 6(6):992-999

34. Mazzulla S, Schella A, Gabriele D et al (2015) Oxidation of human red blood cells by a free radical initiator: effects on rheological properties. Clin Hemorheol Microcirc 60(4):375-388

35. Wozniak B, Wozniak A, Mila-Kierzenkowska C, Kasprzak HA (2016) Correlation of Oxidative and Antioxidative Processes in the Blood of Patients with Cervical Spinal Cord Injury. Oxid Med Cell 
Longev 2016:6094631

36. Nightingale TE, Bhangu GS, Bilzon JLJ, Krassioukov AV (2020) A cross-sectional comparison between cardiorespiratory fitness, level of lesion and red blood cell distribution width in adults with chronic spinal cord injury. J Sci Med Sport 23(2):106-111

37. Rokicki W, Zalejska-Fiolka J, Pojda-Wilczek D, Kabiesz A, Majewski W (2016) Oxidative stress in the red blood cells of patients with primary open-angle glaucoma. Clin Hemorheol Microcirc 62(4):369378

38. Huang CT, DeVivo MJ, Stover SL (1990) Anemia in acute phase of spinal cord injury. Arch Phys Med Rehabil 71(1):3-7

39. Rouleau P, Guertin PA (2010) Traumatic and non-traumatic spinal cord-injured patients in Quebec, Canada. Part 2: biochemical profile. Spinal Cord 48(11):819-824

\section{Tables}

Table 1: Perioperative clinical data of syringomyelia of different aetiologies. 


\begin{tabular}{|c|c|c|c|c|}
\hline & $\begin{array}{c}\text { Chiari I } \\
\text { malformation } \\
(\mathrm{n}=106)\end{array}$ & $\begin{array}{l}\text { Revision } \\
(\mathrm{n}=26)\end{array}$ & $\begin{array}{c}\text { PTS } \\
(n=15)\end{array}$ & $\mathrm{P}$ \\
\hline Male & $25(23.58 \%)$ & $7(26.92 \%)$ & $13(86.67 \%)$ & $\begin{array}{c}< \\
0.0001^{a}\end{array}$ \\
\hline Age, years & $48.0 \pm 12.7$ & $47.0 \pm 11.3$ & $50.5 \pm 8.4$ & $0.8018^{\mathrm{b}}$ \\
\hline $1-20$ & $5(4.71 \%)$ & 0 & 0 & \\
\hline $20-29$ & $3(2.83 \%)$ & $3(11.54 \%)$ & 0 & \\
\hline $30-39$ & $14(13.21 \%)$ & $4(15.38 \%)$ & $1(6.67 \%)$ & \\
\hline $40-49$ & $29(27.36 \%)$ & $5(19.23 \%)$ & $6(40 \%)$ & \\
\hline $50-59$ & $36(33.96 \%)$ & $11(42.31 \%)$ & $5(33.33 \%)$ & \\
\hline $60+$ & $19(17.92 \%)$ & $3(11.54 \%)$ & $3(20 \%)$ & \\
\hline Previous ASIA & & & & $0.001^{\mathrm{c}}$ \\
\hline Complete & NA & 0 & $6(40 \%)$ & \\
\hline Incomplete & & $26(100 \%)$ & $9(60 \%)$ & \\
\hline \multicolumn{5}{|l|}{ Previous surgery } \\
\hline Yes & NA & $26(100 \%)$ & $9(60 \%)$ & \\
\hline Conservative & & 0 & $6(40 \%)$ & \\
\hline Interval & NA & $61.7 \pm 60.4$ & $203.0 \pm 136.4$ & $0.0004^{\mathrm{d}}$ \\
\hline $\begin{array}{l}\text { SM Symptom duration, } \\
\text { months }\end{array}$ & $71.5 \pm 94.3$ & $88.9 \pm 85.5$ & $32.3 \pm 48.9$ & $0.0173^{b}$ \\
\hline$<1$ & $4(4.08 \%)$ & 0 & $1(6.67 \%)$ & \\
\hline $1-6$ & $22(22.45 \%)$ & $1(3.85 \%)$ & $4(26.67 \%)$ & \\
\hline $7-24$ & $25(25.51 \%)$ & $6(23.08 \%)$ & $7(46.66 \%)$ & \\
\hline$>24$ & $47(47.96 \%)$ & $19(73.07 \%)$ & $3(20 \%)$ & \\
\hline First SM Sign & & & & $0.258^{\mathrm{c}}$ \\
\hline Neuropathic pain & $32(32.65 \%)$ & $11(42.31 \%)$ & $2(13.33 \%)$ & \\
\hline Dysesthesia & $37(37.75 \%)$ & $11(42.31 \%)$ & $9(60 \%)$ & \\
\hline Sensory deficit & $9(9.18 \%)$ & 0 & 0 & \\
\hline Motor & $20(20.42 \%)$ & $4(15.38 \%)$ & $4(26.67 \%)$ & \\
\hline \multicolumn{5}{|l|}{ Symptoms } \\
\hline Occipital pain & $31(29.24 \%)$ & $\begin{array}{c}12 \\
(46.15 \%)\end{array}$ & 0 & $0.099^{a}$ \\
\hline Neuropathic pain & $59(55.66 \%)$ & $\begin{array}{c}19 \\
(73.08 \%)\end{array}$ & 7(46.67\%) & $0.178^{\mathrm{a}}$ \\
\hline Dysesthesia & $75(70.75 \%)$ & $22(84.62 \%)$ & $12(80 \%)$ & $0.303^{\mathrm{a}}$ \\
\hline Hypesthesia & $61(57.55 \%)$ & $22(84.62 \%)$ & $13(86.67 \%)$ & $0.006^{a}$ \\
\hline Motor power & $50(47.17 \%)$ & 18(69.23\%) & 13(86.67\%) & $0.004^{a}$ \\
\hline Gait & $31(29.24 \%)$ & $14(53.85 \%)$ & 13(86.67\%) & $<0.0001^{\mathrm{a}}$ \\
\hline Sphincter function & $\begin{array}{l}9(8.49 \%) \\
\text { Page 13/21 }\end{array}$ & $3(11.54 \%)$ & $10(60 \%)$ & $<0.0001^{\mathrm{c}}$ \\
\hline
\end{tabular}


Swallowing function

Sweating

ASIA

UE

LE

PP

LT

A

B

C

D

E

Radiological data

Ventilation dilation

Scoliosis

Occipitalization of atlas

Basilar invagination

Klippel-Feil syndrome

Syringomyelia

Lesion location

Medulla oblongata

Cervical

Cervicothoracic

Thoracic

Thoracolumbar

CTL

Whole

Cord/canal

A
B
C
D
E

Management

Arachnoid opened

Tonsil manipulated

Fusion

Arachnoid lysis

Laminectomy

Arachnoid

$\begin{array}{lccc}11(10.38 \%) & 9(34.62 \%) & 2(13.33 \%) & 0.01^{\mathrm{c}} \\ 14(13.21 \%) & 2(7.69 \%) & 2(13.33 \%) & 0.844^{\mathrm{c}}\end{array}$

$\begin{array}{cccc}48.2 \pm 5.4 & 47.7 \pm 2.8 & 47.7 \pm 3.5 & 0.1012^{\mathrm{b}} \\ 48.8 \pm 5.4 & 48.3 \pm 3.3 & 39.9 \pm 14.2 & <0.0001^{\mathbf{b}} \\ 104.5 \pm 12.2 & 105.3 \pm 7.9 & 91.5 \pm 11.5 & \mathbf{0 . 0 0 0 5}^{\mathbf{b}} \\ 106.1 \pm 11.4 & 105.3 \pm 7.8 & 94.0 \pm 10.1 & <^{\mathbf{0 . 0 0 0 1}} \mathbf{b} \\ 0 & 0 & 0 & \mathbf{0 . 0 0 3}^{\mathbf{b}} \\ 1(0.94 \%) & 0 & 1(6.67 \%) & \\ 1(0.94 \%) & 0 & 4(26.67 \%) & \\ 90(84.91 \%) & 25(96.15 \%) & 9(60 \%) & \\ 14(13.21 \%) & 1(3.85 \%) & 1(6.67 \%) & \end{array}$

7(6.60\%)

$1(3.85 \%)$

0

$0.945^{\mathrm{a} 1}$

$22(20.75 \%)$

5 (19.23\%)

3(13.33\%)

$0.984^{\mathrm{a}}$

8(7.55\%)

3(11.54\%)

0

$0.792^{\mathrm{a} 1}$

$12(11.32 \%)$

$5(19.23 \%)$

0

$0.452^{\mathrm{a} 1}$

$3(2.83 \%)$

0

0

$1.00^{\mathrm{c}}$

106(100\%)

26(100\%)

15(100\%)

2(1.89\%)

0

14(13.21\%)

3 (11.54\%)

2(13.33\%)

90(84.91\%)

1(0.94\%)

0

1(0.94\%)

0

23(88.46\%)

8(46.67\%)

0

2(13.33\%)

0

1(6.67\%)

0

4(20\%)

0

0

$0.009^{b}$

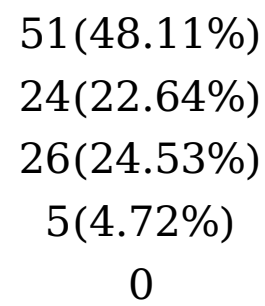

$19(73.08 \%) \quad 11(73.34 \%)$

6(23.08\%) 2(13.33\%)

$1(3.84 \%) \quad 2(13.33 \%)$

0

0

0

0

\section{6(100\%) 26(100\%)}

61(57.55\%) 18(69.23\%)

$0.276^{\mathrm{a}}$

NA

$4(26.67 \%)$

$4(26.67 \%)$

$1(6.67 \%)$

$1(6.67 \%)$ 
lysis+Syringostomy

Arachnoid lysis+Cord

$3(20 \%)$

transection

Cord transection

SSS

Intradural findings

0

1

2

$\begin{array}{cccc} & & 3(20 \%) & \\ & & 1(6.67 \%) & \\ & & 1(6.67 \%) & \\ & & & <0.0001^{b} \\ 14(13.21 \%) & 2(7.69 \%) & 2(16.67 \%) & \\ 89(83.96 \%) & 19(73.08 \%) & 0 & \\ 3(2.83 \%) & 5(19.23 \%) & 10(83.33 \%) & \\ & & & \\ 2(1.89 \%) & 0 & 1(6.67 \%) & \\ 5(4.71 \%) & 5(19.23 \%) & 0 & \\ 1(0.94 \%) & 1(3.84 \%) & 0 & \\ 0 & 0 & 0 & \\ 2(1.89 \%) & 0 & 0 \\ 0 & 0 & 0 \\ 0 & 0 & 0 \\ 0 & 0 & 0 \\ 0 & 0 & 0 \\ 0 & 0 & 1(6.67 \%) & \\ 10(9.43 \%) & 6(23.07 \%) & 2(13.33 \%) \\ 20.7 \pm 6.2 & 21.7 \pm 8.8 & 34.8 \pm 19.4 & \end{array}$

\section{Complication}

Isolated fever

Aseptic meningitis

CSF fistula

Hydrocephalus

Cerebral infarction

Swallowing dys

Haemorrhage

Wound infection

Urinary tract infection Pneumonia

Total

${ }^{*}$ Significant difference $(\mathrm{p}<0.05)$ between groups.

${ }^{\mathrm{a}}$ Chi square test. ${ }^{\mathrm{a} 1}$ Correction of the Chi square test.

$\mathrm{b}_{\text {Kruskal-Wallis test. }}$

${ }^{\mathrm{C}}$ Fisher exact test.

$\mathrm{d}_{\text {Mann-Whitney test. }}$

ene-way ANOVA test.

Table 2: Changes in related symptoms of syringomyelia caused by different aetiologies 


\section{\begin{tabular}{c}
\hline Symptom \\
Occipital pain
\end{tabular}}

Preop

$3 \mathrm{mos}$

$1 \mathrm{yr}$

Neuropathic pain

$\begin{array}{cc}\text { Preop } & 4.1 \pm 0.9 \\ \text { Postop } & 4.2 \pm 0.9 \\ 3 \mathrm{mos} & 4.4 \pm 0.8 \\ 1 \mathrm{yr} & 4.4 \pm 0.8\end{array}$

\section{Dysesthesia}

Preop

Postop

3 mos

$1 \mathrm{yr}$

\section{Hypesthesia}

Preop
Postop
3 mos
1 yr

Motor weakness
Preop

Postop

3 mos

$1 \mathrm{yr}$

\section{Gait ataxia}

$$
\text { Preop }
$$

Postop

3 mos

$1 \mathrm{yr}$

\section{Bladder function}

Preop
Postop
3 mos
1 yr

\section{Swallowing}

$\begin{array}{ll}\text { Preop } & 4.8 \pm 0.6 \\ \text { Postop } & 4.9 \pm 0.3\end{array}$

$(\mathrm{n}=26)$

$(\mathrm{n}=132)$ 


\begin{tabular}{ccccc}
3 mos & $4.9 \pm 0.3$ & $4.8 \pm 0.5$ & $4.9 \pm 0.4$ & $4.9 \pm 0.2$ \\
$1 \mathrm{yr}$ & $4.9 \pm 0.3$ & $4.7 \pm 0.7$ & $4.9 \pm 0.4$ & $4.9 \pm 0.2$ \\
Overall & & & & \\
Better & $80(75.5 \%)$ & $20(77.0 \%)$ & $100(75.7 \%)$ & $9(60.0 \%)$ \\
Unchanged & $13(12.3 \%)$ & $2(7.6 \%)$ & $15(11.4 \%)$ & $1(6.7 \%)$ \\
Worsen & $13(12.2 \%)$ & $4(15.4 \%)$ & $17(12.9 \%)$ & $5(33.3 \%)$ \\
\hline
\end{tabular}

* Unless otherwise specified, all values are expressed as the mean \pm SD.

\# Paralysis of the lower extremities indicates the muscle strength of the upper extremities.

Figures

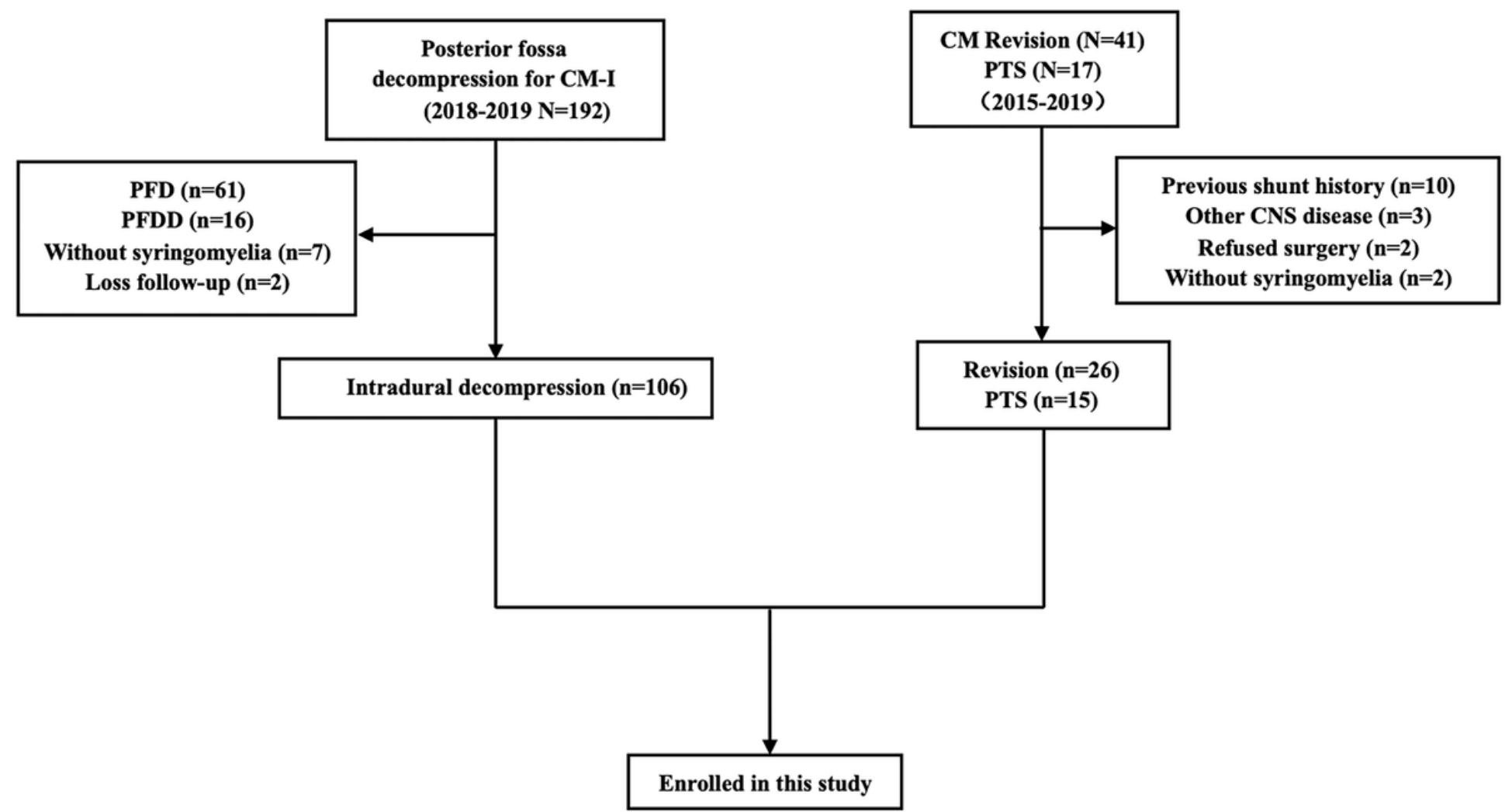

CM-I, Chiari Malformation-I; PTS, post-traumatic syringomyelia; PFD, posterior fossa decompression; PFDD, posterior fossa decompression and duraplasty; CNS, central nervous system;

\section{Figure 1}

Flow chart of 147 consecutive syringomyelia patients between 2015 and 2019. 


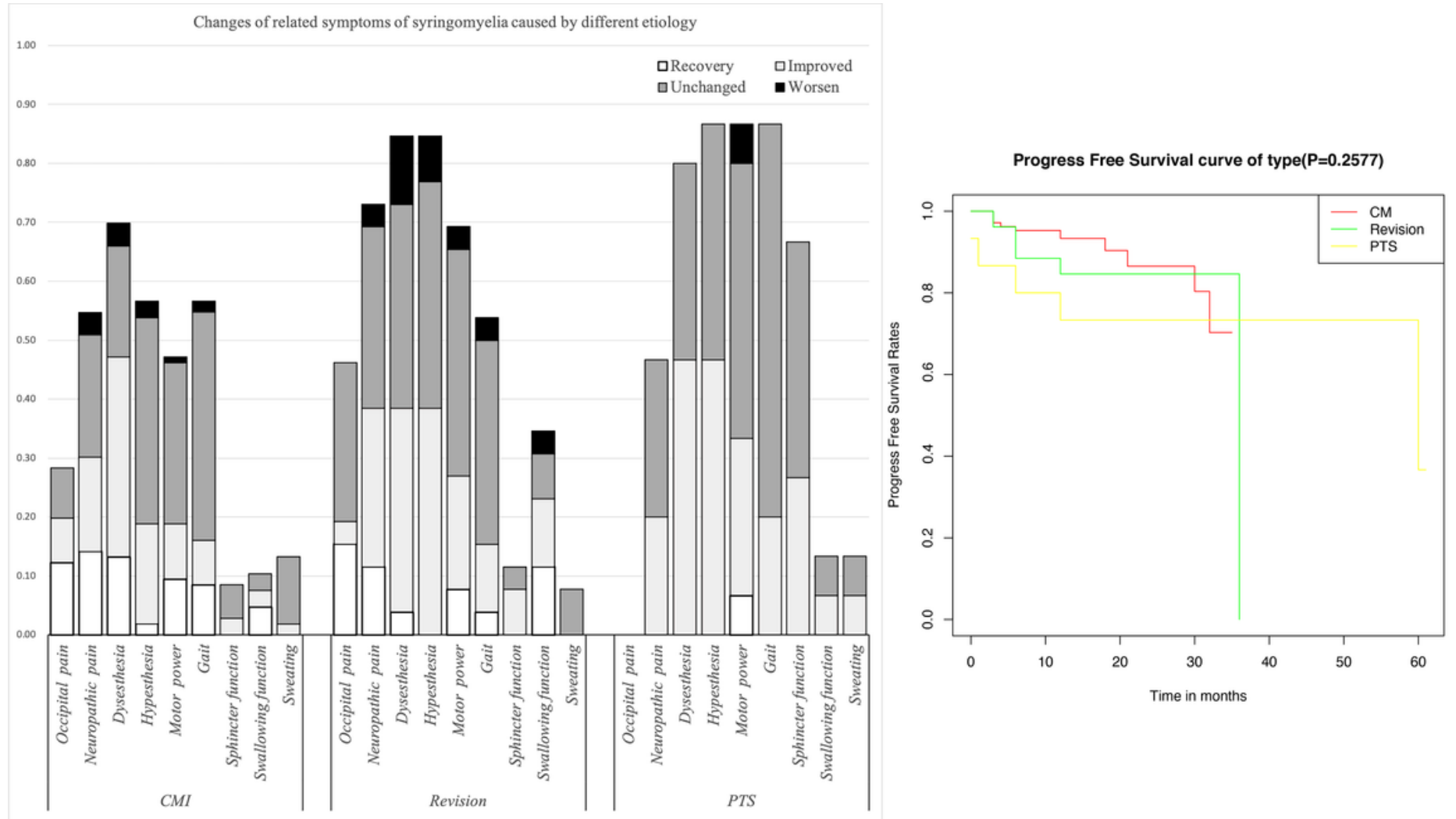

Figure 2

Left chart: Changes in related symptoms of syringomyelia caused by different aetiologies. Right chart: Survival curve of syringomyelia 
Changes in the size of syringomyelia caused by different etiology before and after operation

- $\mathrm{A} \square \mathrm{B} \backsim \mathrm{C} \backsim \mathrm{D} \quad \mathrm{E}$

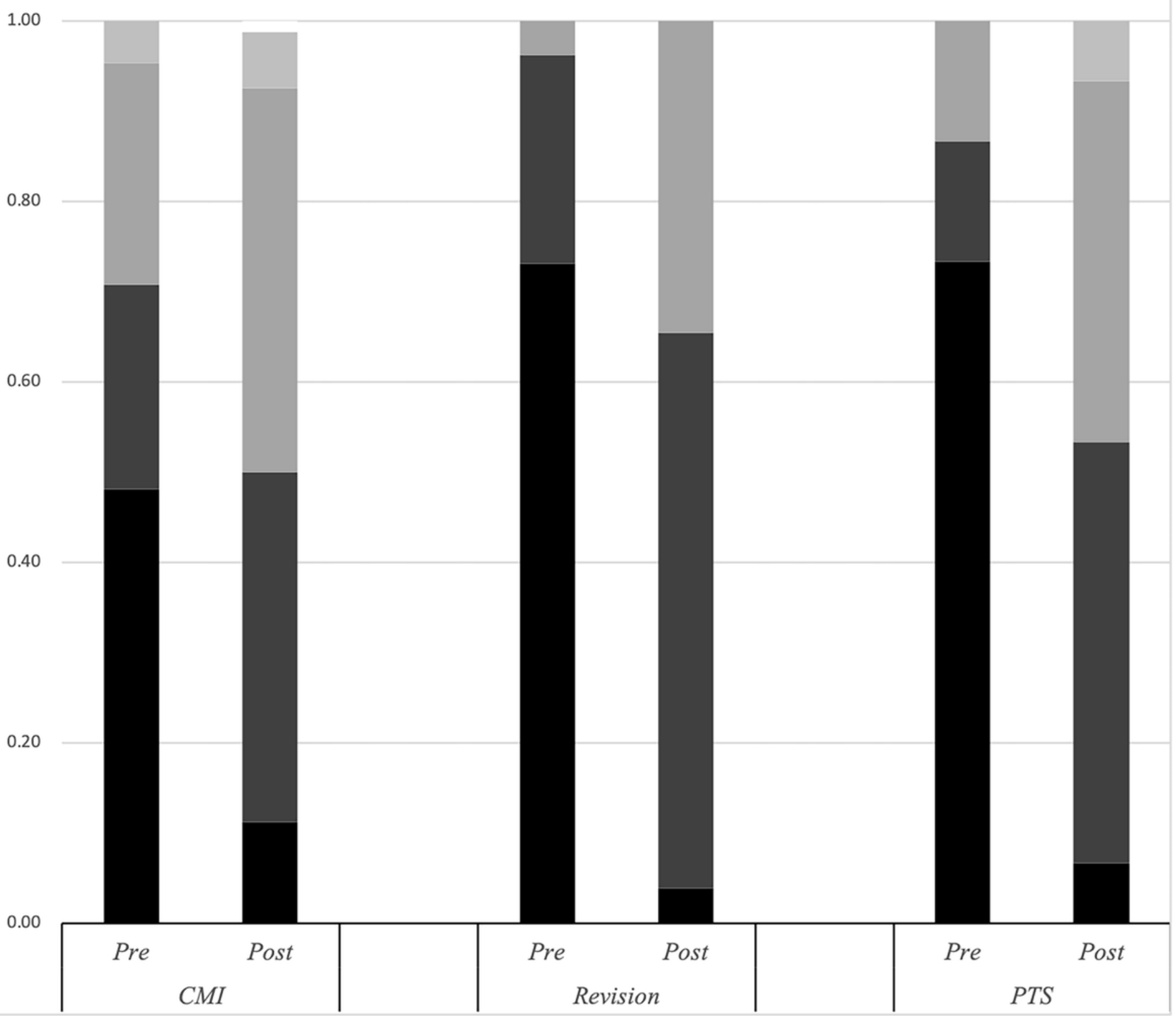

Figure 3

Changes in the size of syringomyelia caused by different aetiologies before and after surgery 

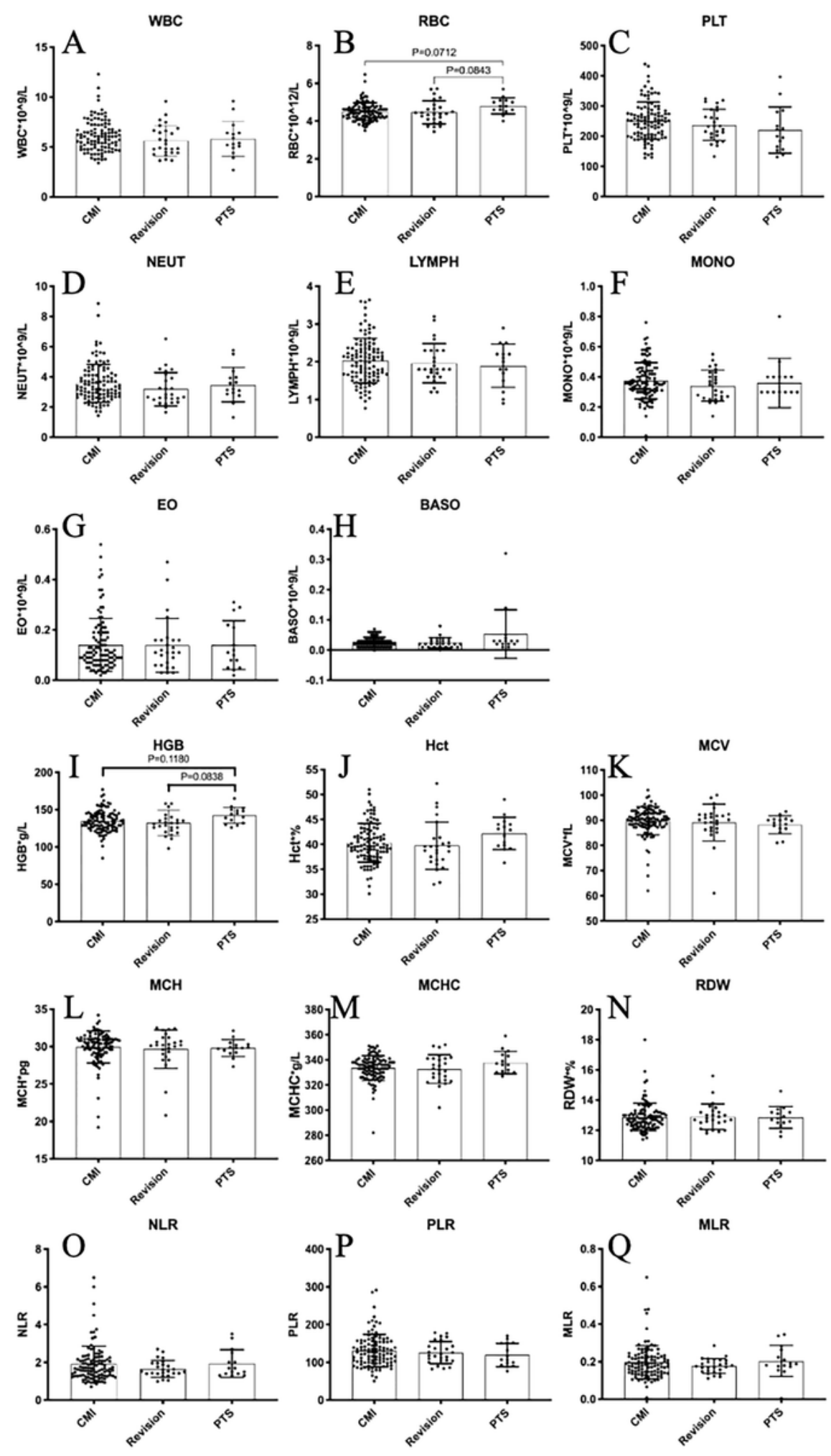

Figure 4

Peripheral blood inflammatory markers of syringomyelia caused by different aetiologies 

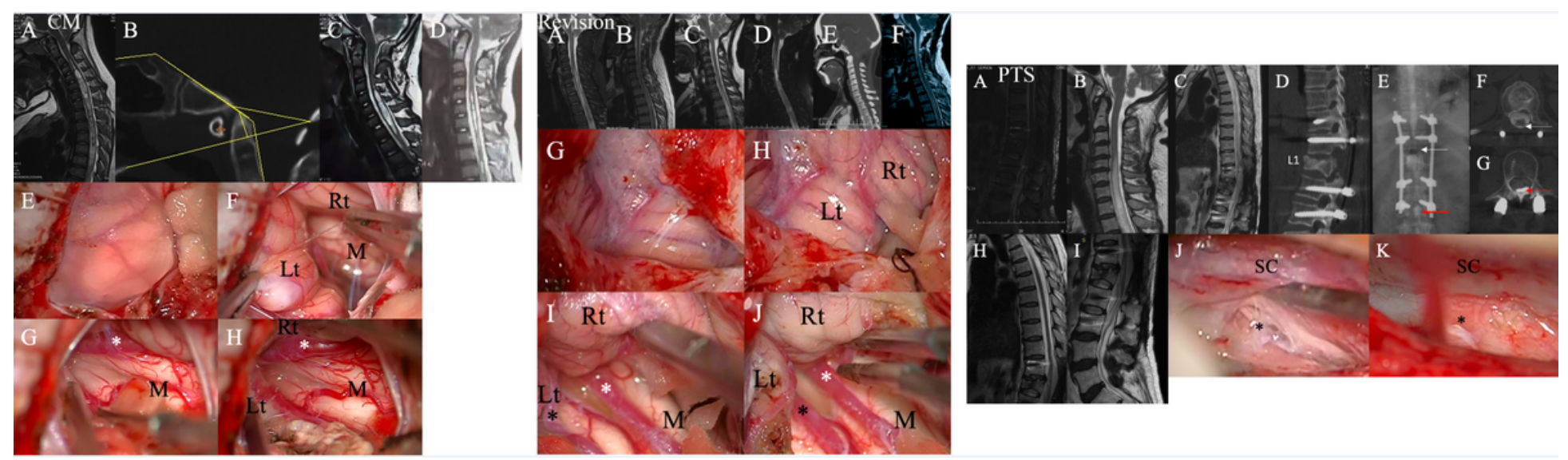

\section{Figure 5}

Left chart: Case presentation of the CM group. A patient was found to have syringomyelia due to facial paralysis. (A) Schematic drawings of the foramen magnum region. Midsagittal T2-weighted MRI scans of the craniocervical region and CT scans suggested that there was no other instability or basilar invagination (B). Three (C) months after the initial surgery and 30 (D) months after the initial surgery showing the ratio of the syrinx/canal from $A$ to $B$. (E, F) A hypertrophic tonsil obstructing the foramen of Magendie. $(G, H)$ The right PICA (asterisk) obstructing the foramen of Magendie was lysed, and the tonsil was coagulated. $\mathrm{Lt}=\mathrm{Left}$ tonsil; $\mathrm{Rt}=$ Right tonsil; $\mathrm{M}=$ Medulla oblongata Middle chart: Case presentation of the revision group. (A) Preoperative sagittal T2-weighted MRI shows a large syringomyelia. (B) Three months, (C) 9 months, and (D) 3 years after the first surgery. (E) CT shows that the partial bone defects of the occipital bone and syringomyelia persisted. (F) Postoperative MRI 2 years after the second surgery showing the syringomyelia was obviously reduced. $(G, H)$ A hypertrophic tonsil obstructing the foramen of Magendie. (I, J) PICA (asterisk) obstructing the foramen of Magendie was lysed, and the tonsil was coagulated. $\mathrm{Lt}=$ Left tonsil; Rt = Right tonsil; $M=$ Medulla oblongata Right chart: Case presentation of the PTS group. (A) A postoperative sagittal T2-weighted MRI scan shows some oedema and internal fixation. (B, C and D): Sagittal T2-weighted MRI and CT after 2 years shows a large syringomyelia (up to C4 and down to the L1 vertebra) and an L1 compression fracture. (E, F and G): Myelography showing that the circulation of cerebrospinal fluid was blocked at L1. ( $\mathrm{H}$ and I): Postoperative sagittal T2-weighted MRI data showing that the syrinx was obviously reduced. ( $\mathrm{J}$ and $\mathrm{K}$ ): Intraoperative images showing that the obvious adhesion around the spinal cord at L1 was removed.

\section{Supplementary Files}

This is a list of supplementary files associated with this preprint. Click to download.

- suptable1.docx

- suptable2.docx

- suptable3.docx 